\title{
Un cadre éthique pour l'échange de données
}

\section{Effy Vayena}

Professeur en bioéthique, Département des sciences de la santé et de technologie, EPF de Zurich

Actuellement, les progrès vertigineux observés à l'interface entre biomédecine et informatique ont pour corollaire l'apparition d'un énorme volume de données relatives à la santé. Ces données sont qualifiées, entre autres, de «big data» du fait de leur étendue et de leur complexité, mais aussi en raison des capacités d'analyse de plus en plus performantes. Ces données sont associées à l'espoir de progrès médicaux majeurs: en effet, elles devraient permettre aux individus de mieux comprendre leur état de santé et de mieux gérer leurs maladies; elles devraient favoriser un dépistage plus précoce des problèmes de santé et permettre une meilleure adaptation des traitements médicaux aux particularités des patients. Par ailleurs, les résultats de traitements peuvent ainsi être plus efficaces, et les coûts réduits. Les découvertes médicales et le développement de médicaments devraient ainsi progresser et même s'accélérer.

Cet immense potentiel lié aux données fait néanmoins face à un obstacle: les données sont encore enregistrées de manière isolée, ce qui empêche leur analyse et finalement freine la marche du progrès scientifique. Cette situation est imputable à plusieurs facteurs, qu'ils soient de nature technique, pratique, réglementaire ou éthique. Le Swiss Personalized Health Network $(S P H N)$ est une initiative nationale [1] destinée à développer les infrastructures nécessaires à l'échange des données et, de ce fait, à mieux utiliser les données relatives à la santé dans une optique de recherche et d'innovation.

Dans le domaine de la biomédecine et de la santé en général, la recherche basée sur des données dépend de l'évolution des standards techniques appropriés, qui assurent un échange sécurisé et un traitement efficace des données. Le SPHN a donc pour objectif principal de développer des normes et standards uniformisés simplifiant l'accès aux données de santé pour les chercheurs suisses. Ces données sont essentielles non seulement pour la Suisse, mais aussi au niveau international. La vision du SPHN consiste à atteindre cet objectif en respectant des standards éthiques stricts.

\section{Quatre principes de base de gestion des données sensibles}

$\mathrm{Vu}$ le caractère sensible des données personnelles et des informations relatives à la santé, un constat s'impose de manière unanime: ces données doivent être

\section{Congrès $\mathrm{H}+2017$ : Homme et technologie - la marche irrésistible du numérique?}

L'évolution technologique et le numérique se développent de manière extrêmement rapide. Le Congrès H+ apportera le 8 novembre 2017 des réponses aux interrogations et mettra en évidence les risques et les avantages des évolutions actuelles et à venir. Le programme du congrès entend couvrir les principaux domaines d'action des hôpitaux et des cliniques et jeter des ponts entre les acteurs. Dans cet esprit, $\mathrm{H}+$ souhaite tout particulièrement stimuler les interactions grâce à des Knowledge Lounges inédites. En lieu et place des exposés et des ateliers, des expertes et des experts des institutions hôtes feront vivre différents aspects du thème du congrès.

- Ecole polytechnique fédérale de Lausanne (EPFL): Data-Driven Intelligence

- ETH Zürich: Smart Technologies

- Commission pour la technologie et l'innovation (CTI): Encouragement de l'innovation dans le domaine des sciences de la vie

ASSM, SPHN et Science \& Cité: Génial le digital aussi éthique et social?

- H+ Bildung et Espace Compétences: La numérisation pousse au développement de l'entreprise

- HINT SA et LA POSTE: Des tendances numériques prometteuses et sûres

L'ISFM accorde 5 crédits pour la participation au congrès $\mathrm{H}+$.

www.hplus-kongress.ch 
traitées avec un soin tout particulier. Le milieu de la recherche doit se pencher sérieusement sur les questions éthiques soulevées tout au long du processus, depuis la recherche du consentement pour la collecte initiale des données jusqu'à l'utilisation de ces données. C'est pourquoi le SPHN s'est doté, dès sa création, d'un groupe de conseillers spécialisés dans les questions éthiques et sociales, qui se consacre à ces aspects: l'Ethical, Legal and Social Implications Advisory Group (ELSIag). Sa première mission a résidé dans l'élaboration d'un cadre éthique garantissant un traitement responsable des données de santé.

\section{Le SPHN a pour objectif de développer des standards uniformisés simplifiant l'accès aux données pour les chercheurs suisses, tout en respectant un cadre éthique stricte.}

Lors de l'élaboration de ce document (publié sur le site Internet du SPHN), l'ELSIag a pris en compte des directives et principes nationaux et internationaux et mené des discussions approfondies, tant parmi ses propres rangs qu'avec d'autres organes du SPHN. L'ELSIag a finalement identifié quatre principes de base constituant la colonne vertébrale normative du traitement responsable de données: respect des personnes impliquées, protection de la vie privée, équité numérique, et engagement responsable.

\section{Respect des personnes impliquées}

Ce principe impose le respect, la protection et la promotion des droits et de la dignité des individus mettant à disposition leurs données de santé dans le cadre de projets de recherche ou de traitements médicaux.

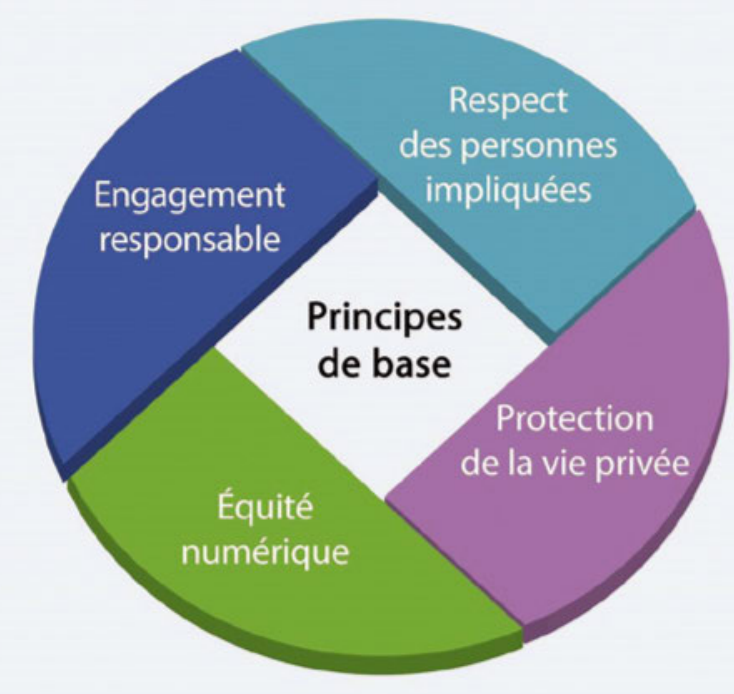

Cadre éthique du SPHN, avec ses quatre principes de base pour un échange responsable des données.
La nécessité d'obtenir le consentement de la personne concernée constitue donc une exigence centrale liée à ce principe pour l'utilisation des données. Entrent ici en ligne de compte les obligations des utilisateurs des données si la personne devait revenir sur son consentement (thème particulièrement complexe dans l'ère du «big data» et dans le contexte d'une mise en réseau constante). Ce principe est en outre pertinent pour les mesures de communication à mettre en œuvre pour la transmission des résultats cliniques des recherches aux participants aux études.

\section{Protection de la vie privée}

Ce principe impose la protection de la sphère privée et la confidentialité des données de santé.

La protection des données est un droit fondamental qui exige une attention particulière. Pour être respecté, ce droit nécessite une série de mesures techniques et administratives. Des directives complémentaires afférentes au cadre éthique viennent souligner l'importance de la sécurité des données et des normes d'anonymisation, et requièrent des contrôles réguliers de l'utilisation des données.

\section{Equité numérique}

En vertu de ce principe, les données pouvant être utilisées à des fins de recherche et les résultats des recherches doivent être mis à disposition pour des travaux ultérieurs afin de favoriser le progrès scientifique dans son ensemble.

Les données relatives à la santé sont une ressource inestimable qui augmentent notre connaissance des maladies et peut contribuer à améliorer la prise en charge médicale. Ce potentiel implique néanmoins une obligation, et plus précisément la nécessité de mettre à disposition les données correspondantes pour une recherche irréprochable sur le plan éthique. Léquité requiert un accès rapide aux données. Aucun accord exclusif empêchant cet accès aux chercheurs ne doit être conclu. En vertu de ce principe, les données doivent être rendues accessibles, notamment pour servir les objectifs du SPHN, et ce sans intérêts financiers.

\section{Engagement responsable}

Ce principe exige un traitement des données équitable, transparent et conforme à la loi.

Tous les collaborateurs traitant les données personnelles à des fins de recherche ont une obligation de rendre compte des conséquences de leurs actes, tant pour les participants aux projets de recherche que pour la société dans son ensemble. Les procédures et mécanismes encadrant l'utilisation des données doivent faire l'objet de contrôles réguliers. Les partici- 
pants aux projets de recherche ont notamment le droit de connaître la manière dont une organisation traite leurs données. Entrent également ici en ligne de compte les conditions selon lesquelles l'organisation permet à des utilisateurs tiers d'accéder aux données. La transparence est une composante essentielle de l'obligation de rendre compte.

\section{Importance sur le plan pratique}

Sur la base de ces quatre principes fondamentaux, le cadre éthique doit être considéré comme un ensemble dans lequel toutes les exigences doivent être satisfaites

\section{La protection des données est un droit fonda- mental qui exige une attention particulière.}

en même temps. Ce n'est qu'au prix de l'interaction de ces principes (et non de l'application de principes isolés) que des résultats optimaux sont garantis. Des directives spécifiques issues de ces principes de base doivent faciliter leur mise en œuvre synergétique. En outre, ce cadre n'est pas figé; il bénéficiera des échanges entre toutes les parties prenantes et sera enrichi d'autres éléments dans le sillage des exigences croissantes applicables aux réseaux.

Les hôpitaux recueillent et utilisent d'importants volumes de données de santé. En qualité de partie prenante majeure dans le domaine de la santé, ils bénéficient de la confiance de leurs patients et contribuent à l'application et au développement de l'utilisation responsable des données de santé. Le cadre éthique peut jouer le rôle de socle commun pour l'élaboration de règles de gouvernance et renforcer le rôle des hôpitaux en tant qu'administrateur des données de santé. Le cadre doit notamment garantir que l'initiative du SPHN est conforme aux standards applicables en matière d'éthique, qu'elle promeut les droits, les intérêts et le bien-être des participants aux projets de recherche, qu'elle permet le développement efficace de précieuses connaissances et la confiance du public dans l'activité du réseau. Toutefois, ce cadre n'a pas valeur de prescription. Il constitue plutôt un code commun servant notre intérêt collectif en tant que société.

Le SPHN offre l'occasion d'améliorer, de manière fondamentale, la culture de la recherche en rapport avec les données de santé. Il s'agit d'un objectif que nous partageons avec de nombreux autres pays. Cependant, la Suisse occupe une position unique pour aller de l'avant. Outre la recherche biomédicale et bioinformatique habituellement de grande qualité, on observe de nombreuses autres tendances convergentes pouvant stimuler le développement de la recherche utilisant les données de santé: parmi ces dernières, citons l'introduction du dossier électronique du patient, l'élaboration du formulaire général de consentement pour la réutilisation des données (modèle de consentement général [2]) ainsi que les multiples possibilités d'encouragement des projets de recherche dans le domaine de la numérisation et des technologies apparentées. Tous ces éléments sont autant d'indicateurs importants pour un intérêt national fort et un engagement marqué dans ces domaines. Si nous parvenons à être motivés par ces développements sur la base de principes éthiques solides, cela peut influer sur la manière dont nous menons nos recherches, et améliorons, sur le long terme, la santé de chaque individu et de la population en général.

\section{Références}

1 Voir également: Lawrence A, Selter L. Swiss Personalized Health Network (SPHN): Die nationale Initiative im Überblick. Bull méd suisses 2017;98(19):595-6.

2 Voir le modèle de CG 1/2017 de l'Académie Suisse des Sciences Médicales (ASSM) et des commissions d'éthique suisses pour la recherche sur l'être humain (swissethics), consultable à l'adresse suivante: assm.ch/consentement-general

\section{Crédit illustration}

(c) Effy Vayena / showeet.com 